\title{
Crisis epiléptica como primera manifestación de sarcoidosis. Caso clínico
}

\author{
CRISTINA LIDIA FERNÁNDEZ-REY, ANTONIO SAIZ AYALA, \\ MARÍA JESÚS ÁLVAREZ-URÍA TEJERO, ELENA SANTAMARTA LIÉBANA, \\ SERAFÍN COSTILLA GARCÍA
}

\section{Sarcoidosis presenting as partial seizures. Report of one case}

The nervous system is affected in 10\% of patients with sarcoidosis. However, neurological disturbances are rarely the first manifestation of the disease. We report a 36-year-old woman presenting with partial seizures that generalized secondarily. Magnetic resonance showed a left parietal cortical-subcortical lesion with a minimal mass effect, moderate vasogenic edema and intense enhancement with intravenous contrast. A magnetic resonance spectroscopy disclosed a low aggressiveness profile, compatible with an inflammatory lesion. Angiotensin converting enzyme levels were normal. The lesion was excised and the pathological study showed the presence of granulomas with dubious necrosis. The patient was treated with antituberculous drugs. One year later, the lesion had grown and a thorax CT scan showed numerous mediastinal and hilar lymphadenopathies. A new determination of angiotensin converting enzyme disclosed elevated levels and the biopsy of mediastinal lymph nodes confirmed the presence of sarcoidosis.

(Rev Med Chile 2010; 138: 473-477).

Key words: Peptidyl-dipeptidase A; Sarcoidosis; Seizures.

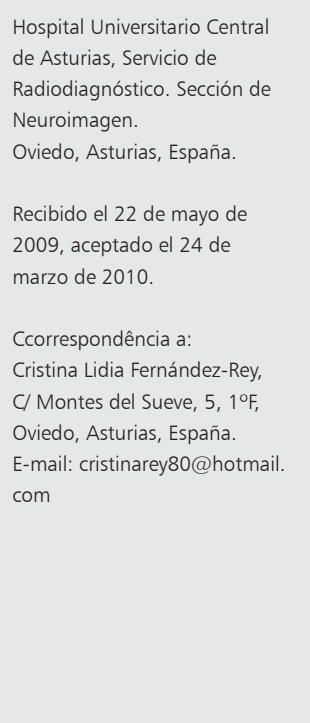

$\mathrm{E}$ 1 compromiso neurológico como forma de presentación de la sarcoidosis en ausencia de enfermedad sistémica constituye una rareza. En estos casos existe una gran dificultad diagnóstica dada la inespecificidad de los síntomas clínicos y de los hallazgos en las pruebas de imagen. Sin embargo, una evaluación global de los hallazgos radiológicos, incluyendo la espectroscopia RM, junto con el contexto clínico del paciente permite reducir el espectro de diagnósticos diferenciales y sospechar la enfermedad.

\section{Caso clínico}

Mujer de 36 años con antecedentes de anorexia y bulimia que ingresó en el Servicio de Neurología tras dos episodios de crisis parciales simples con generalización secundaria. Las crisis comenzaban con parestesias y adormecimiento del miembro superior derecho y continuaban con movimientos tónico-clónicos generalizados y pérdida del conocimiento.

En la tomografía computarizada (TC) craneal realizada se evidenció una masa intracerebral a nivel parietal parasagital izquierdo y el electroencefalograma demostró una asimetría del ritmo de base con presencia de alteraciones funcionales en el hemisferio izquierdo.

Desde el ingreso la paciente presentaba unas exploraciones general y neurológica normales. Las pruebas analíticas, incluyendo pruebas de serología, microbiología y punción lumbar fueron negativas.

Se instauró tratamiento con corticoides que consiguió una disminución llamativa del edema vasogénico que acompañaba a la lesión. A continuación se realizó una resonancia magnética $(\mathrm{RM})$ y un estudio de espectroscopia-RM cuyos resultados 
Crisis epiléptica como primera manifestación de sarcoidosis. Caso clínico - C.L. Fernández-Rey et al

señalaban una naturaleza benigna de la lesión, y un probable origen inflamatorio tipo granulomatoso.

Posteriormente, se realizó exéresis parcial de la tumoración, con resultado histológico de granulomas con dudosa necrosis. Los niveles séricos de la enzima convertidora de la angiotensina fueron normales y las pruebas de imagen complementarias (radiografía de tórax y ecografía abdominal) no mostraron hallazgos patológicos. Se decidió entonces administrar un tratamiento tuberculostático empírico, a la espera de resultados microbiológicos definitivos.

En el seguimiento realizado un año después, se observó un lento crecimiento de la lesión. Por otra parte, no se demostró la existencia de micobacterias tras el análisis microbiológico y las crisis comiciales persistían con periocidad trimestral a pesar de haber recibido varios tratamientos (ácido valproico, levetiracetam y clobazam).

Se solicitó entonces una radiografía de tórax apreciándose la existencia de ensanchamiento mediastínico y crecimiento hiliar bilateral. Ante estos hallazgos se realizó TC toraco-abdominal, demostrándose múltiples adenopatías hiliares bilaterales y mediastínicas. En el parénquima pulmonar no se

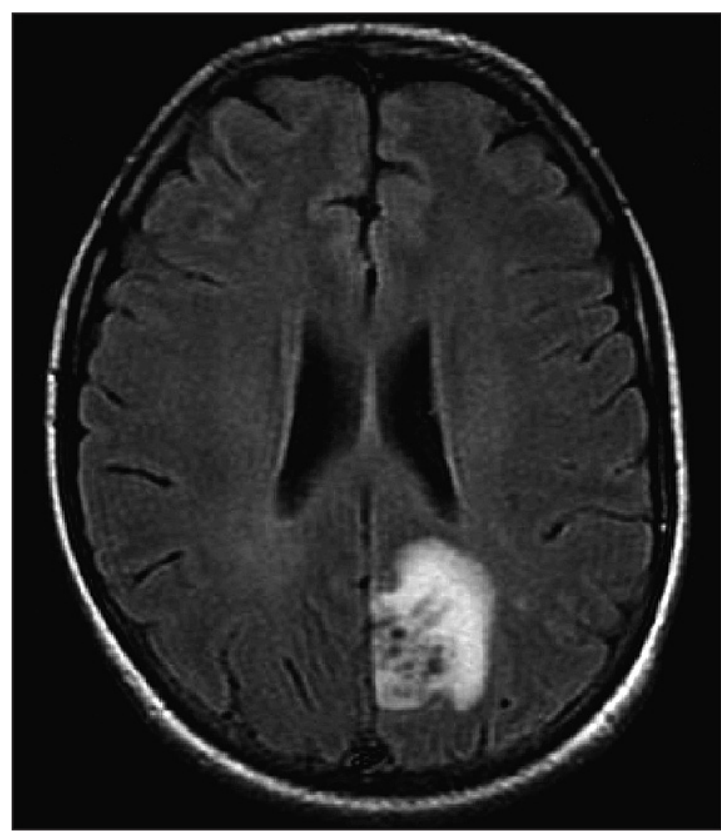

Figura 1. RM corte axial, secuencia T2 FLAIR, que demuestra una lesión parasagital parietal izquierda con moderado edema vasogénico asociado. observaron signos de engrosamiento intersticial. Ante la sospecha de sarcoidosis se realizó una nueva determinación de los niveles en plasma de la enzima convertidora de la angiotensina demostrándose una elevación de los mismos.

La biopsia de las adenopatías mediastínicas confirmó el diagnóstico de sarcoidosis pulmonar estadio I.

Se concluyó por tanto, que la lesión cerebral había sido la primera manifestación de la enfermedad de sarcoidosis en esta paciente y se instauró tratamiento con corticoesteroides. La evolución posterior fue favorable.

\section{Hallazgos radiológicos}

La resonancia magnética cerebral demostró la existencia de una lesión parasagital parietal izquierda, córtico-subcortical, con mínimo efecto de masa y moderado edema perilesional asociado (Figura 1). En las imágenes obtenidas tras la administración de contraste endovenoso (Figura 2) se observó un realce llamativo de la lesión con áreas de captación anular. Aunque no se identificó un claro realce de la duramadre, sí se apreció

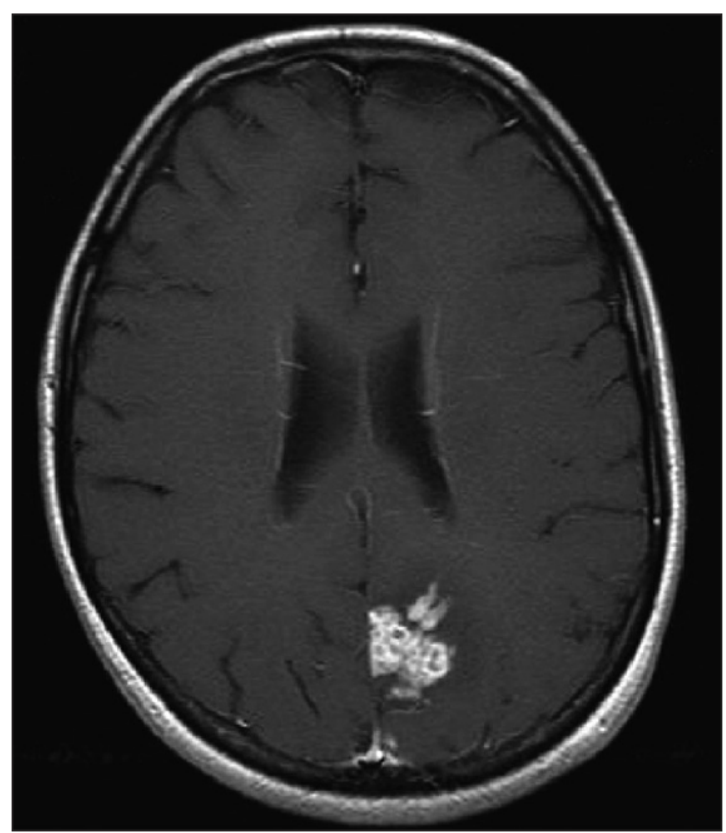

Figura 2. RM corte axial, secuencia $T 1$ con Gadolinio, muestra una captación de contraste intensa e irregular con pequeños focos de realce anular en el seno de la lesión y captación leptomeníngea. 
una participación y captación de la leptomeninge adyacente a las lesiones.

Se completó el estudio de imagen con RM espectroscopia (Figura 3), donde se demostró una discreta elevación de los niveles de colina (Cho) y una mínima disminución del $\mathrm{N}$-acetil-aspartato (NAA), lo que indicaba una baja agresividad de la lesión.

El aspecto de las lesiones y el estudio de espectroscopia señalaban un origen inflamatorio tipo granulomatoso, muy sugestivo de neurosarcoidosis.

En la radiografía de tórax y el TC torácico realizados dos años después se evidenció la aparición de adenopatías hiliares y mediastínicas (Figura 4).

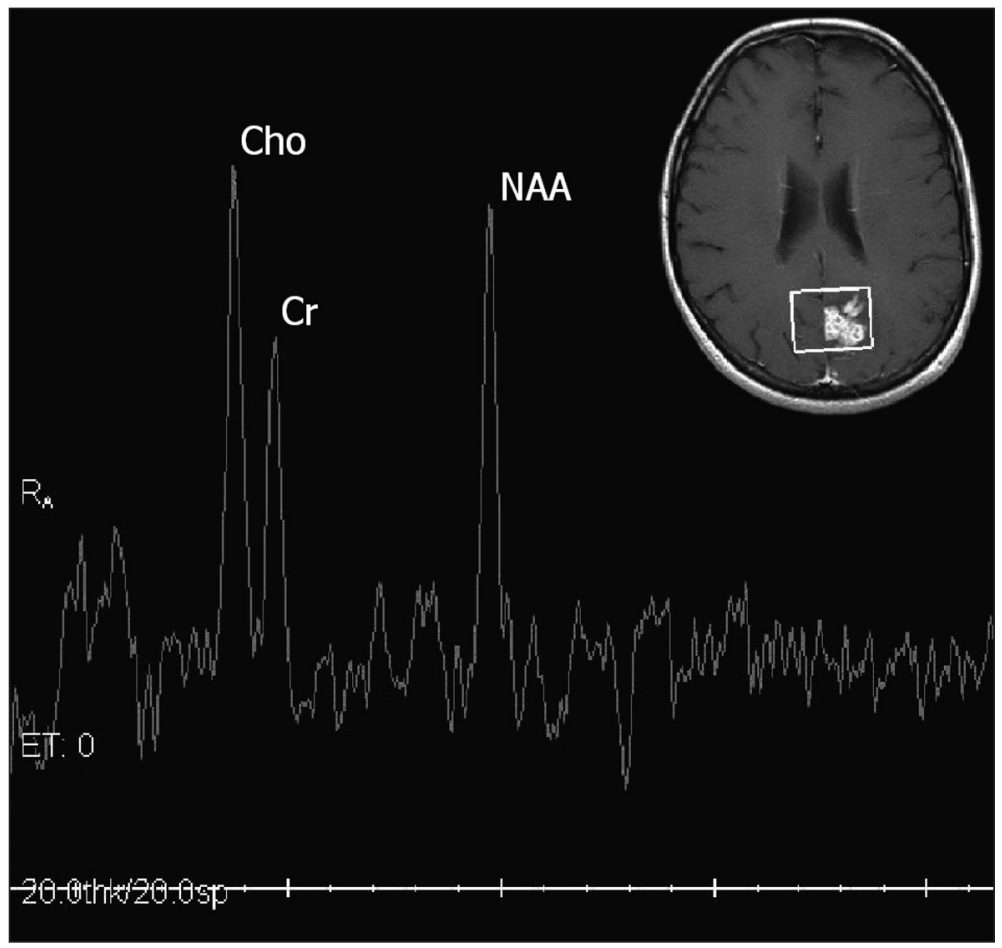

Figura 3. Estudio de espectroscopia RM que demuestra una discreta elevación de los niveles de colina (Cho) y una mínima disminución de la señal de $\mathrm{N}$-acetil-aspartato (NAA). Índice colina-creatina (Cho/ $\mathrm{Cr})<2$. Hallazgos sugestivos de poca agresividad que apoyan el diagnóstico de proceso granulomatoso y hacen improbable un origen neoplásico maligno.
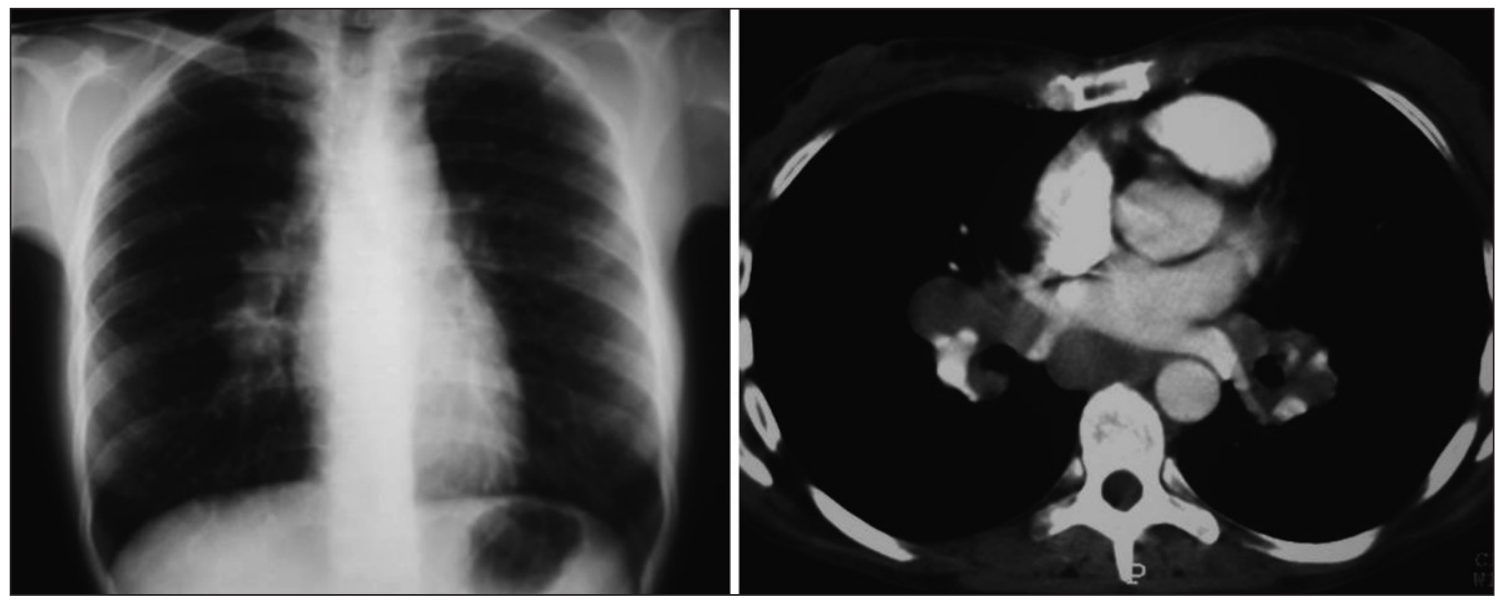

Figura 4. Radiografía simple de tórax postero-anterior e imagen axial de TC torácico a nivel de los hilios mostrando adenopatías hiliares bilaterales y subcarinal. 


\section{Discusión}

La sarcoidosis es una enfermedad multisistémica de tipo inflamatorio e inmunológico y causa desconocida, que afecta al sistema nervioso central en 5-15\% de las ocasiones. Se ha estimado que la afectación del sistema nervioso central en ausencia de enfermedad sistémica ocurre tan sólo en $1 \%$ de los casos ${ }^{1}$.

La clínica de la neurosarcoidosis va a depender de la localización de las lesiones. Generalmente, causa déficit neurológico focal y afectación de los pares craneales, aunque hasta en $50 \%$ de las ocasiones los pacientes están asintomáticos. La parálisis del facial como resultado de infiltración granulomatosa es el síntoma neurológico más común ${ }^{2}$. Otros síntomas frecuentes son la pérdida de visión, cefalea, convulsiones y signos de irritación meníngea ${ }^{1}$. La localización supraselar es bastante característica y se asocia a disfunciones del hipotálamo e hipófisis. La afectación de la médula espinal es infrecuente y se manifiesta clínicamente como debilidad de miembros inferiores y otros síntomas inespecíficos de mielopatía.

El diagnóstico definitivo de la enfermedad se realiza mediante una biopsia que confirme la presencia de granulomas no caesificantes. En ocasiones, la localización de las lesiones es poco accesible para realizar una biopsia y las pruebas de imagen adquieren un papel esencial. El análisis de los niveles séricos del enzima convertidor de angiotensina también puede resultarnos útil. Sin embargo, debemos recordar que el enzima se eleva en un porcentaje variable de los casos, oscilando entre $5 \%$ y $50 \%$ de los pacientes con neurosarcoidosis ${ }^{1}$. Asimismo, debemos tener en cuenta, que el enzima también se eleva en otras enfermedades, tales como, la diabetes, silicosis, y cirrosis ${ }^{1}$.

Respecto al tratamiento está indicada la administración de corticoesteroides a altas dosis al inicio de los síntomas. En los casos más severos o en las recurrencias pueden ser necesarios otros fármacos inmunosupresores ${ }^{2,3}$.

El espectro de anomalías cerebrales asociadas a neurosarcoidosis es amplio y variado ${ }^{3}$, pudiendo afectarse cualquier parte del sistema nervioso central. Existe una predilección por la afectación de la leptomeninge, en especial las cisternas de la base con infiltración de los pares craneales; sin embargo, la afectación del parénquima o de la duramadre también es posible.
La presencia de enfermedad parenquimatosa se manifiesta en $45-50 \%$ de los casos como lesiones inespecíficas de la sustancia blanca que no realzan tras la administración de contraste $e^{4,5}$. Estas lesiones presentan un aspecto similar a la enfermedad de sustancia blanca de origen vascular (isquemia, hipertensión).

Otras formas de afectación del parénquima consisten en lesiones nodulares hipercaptantes de contraste. La afectación multifocal se encuentra hasta en $35 \%{ }^{3,4}$, mientras que la infiltración parenquimatosa aislada ocurre en $9-10 \%$ de las ocasiones $^{4}$. En estos casos el diagnóstico puede ser complejo ya que por su aspecto simulan neoplasias, siendo necesaria la biopsia quirúrgica en muchas ocasiones ${ }^{5}$.

La presencia de lesiones afectando a la duramadre constituye otras formas de presentación característica de la neurosarcoidosis ${ }^{4,6}$. En estos casos los hallazgos por imagen pueden resultar indistinguibles de los presentes en los meningiomas o en los linfomas, e incluso en otras formas menos frecuentes de engrosamiento dural como la meningitis crónica o la paquimeningitis hipertrófica ${ }^{6}$.

Finalmente, el compromiso de las leptomeninges es el hallazgo más característico. Ocurre hasta en $40 \%$ de los casos de neurosarcoidosis y es fácilmente detectable en las imágenes obtenidas tras la administración de gadolinio ${ }^{4,6,7}$. Se puede observar un engrosamiento del espacio subaracnoideo, con realce difuso o nodular. Puede existir diseminación a través de los espacios perivasculares simulando infiltración del parénquima ${ }^{1,6,8}$.

La hidrocefalia puede ocurrir como resultado de la inflamación e infiltración de las meninges y del espacio subaracnoideo, o menos frecuentemente como resultado de compresión del acueducto de silvio o del cuarto ventrículo ${ }^{6}$.

La prueba de imagen de elección en el diagnóstico y seguimiento de la neurosarcoidosis es la $\mathrm{RM}$, fundamentalmente las imágenes obtenidas tras la administración de gadolinio ${ }^{6,7,9,10}$. Los hallazgos por imagen obtenidos en el caso clínico presentado aunque no son patognomónicos, nos permiten orientar el diagnóstico hacia una enfermedad de tipo inflamatorio. La presencia de una lesión cerebral parenquimatosa en una paciente joven, con probable participación de la leptomeninge debe incluir la neurosarcoidosis 
entre las posibles opciones diagnósticas. La respuesta rápida y eficaz al tratamiento con córticoesteroides apoya este diagnóstico. Sin embargo, también debemos considerar otros diagnósticos, tales como, la tuberculosis, infección por hongos, e incluso neoplasias como el linfoma o los gliomas. El estudio de espectroscopia RM demuestra niveles de colina discretamente elevados, con una relación colina/creatina $(\mathrm{Cho} / \mathrm{Cr}$ ) inferior a 2, que junto con la mínima disminución del $\mathrm{N}$-acetil-aspartato sugieren una lesión de baja agresividad, probablemente de tipo inflamatorioinfeccioso. Sin embargo, el papel fundamental de la RM radica en confirmar el diagnóstico en pacientes con sospecha clínica de sarcoidosis y datos de afectación sistémica ${ }^{7}$.

Por otra parte, debemos destacar el papel de las pruebas de imagen en el seguimiento y control de la enfermedad, ya que existe un alto índice de progresión y recurrencia.

El caso presentado es extremadamente atípico no sólo por la inicial afectación cerebral en ausencia de afectación sistémica, sino por la existencia de una lesión única hipercaptante de contraste que fácilmente podría confundirse con un proceso maligno.

La evolución clínica, los estudios histológicos y microbiológicos y las pruebas de imagen confirmaron el diagnóstico de sospecha de neurosarcoidosis.

\section{Referencias}

1. Smith JK, Matheus MG, Castillo M. Imaging Manifestations of Neurosarcoidosis. Am J Roentgenol 2004; 182: 289-95.

2. Nowak DA, Widenka DC. Neurosarcoidosis: a review of its intracranial manifestations. J Neurol 2001; 248: 36372.

3. Pettersen JA, Zochodne DW, Bell RB, Martin L, Hill MD. Refractory neurosarcoidosis responding to infliximab. Neurology 2002; 59: 1660.

4. Pickuth D, Spielmann RP, Heywang-Köbrunner SH. Role of radiology in the diagnosis of neurosarcoidosis. Eur Radiol 2000; 10: 941-4.

5. Brinar VV, Habek M. Isolated central nervous system sarcoidosis: a great mimicker. C Neurol Neurosurg 2008; 110: 939-42.

6. Hayes WS, Sherman JL, Stern BJ, Citrin CM, Pulaski PD. MR and CT evaluation of intracranial sarcoidosis. Am J Radiol 1987; 149: 1043-9.

7. Khaw KT, Manji H, Britton J, Schon F. Neurosarcoidosis--demonstration of meningeal disease by gadolinium enhanced magnetic resonance imaging. J Neurol Neurosurg Psychiatry 1991; 54: 499-502.

8. Mirfakhraee M, Crofford MJ, Guinto FC Jr, Nauta HJ, Weedn VW. Virchow-Robin space: a path of spread in neurosarcoidosis. Radiology 1986; 158: 715-20.

9. Christoforidisa GA, Spickler Recio MV, Mehta BM. MR of CNS Sarcoidosis: Correlation of Imaging Features to Clinical Symptoms and Response to Treatment. AJNR Am J Neuroradiol 1999; 20: 655-69.

10. Pickuth D, Heywang-Köbrunner SH. Neurosarcoidosis: evaluation with MRI. J Neurol 2000; 27: 185-8. 